\section{Association of anti-Ro52 autoantibodies with interstitial lung disease in connective tissue diseases}

We read with great interest the article by Sabbagh et al demonstrating that anti-Ro52 autoantibodies were connected with the development of interstitial lung disease (ILD) in patients with juvenile myositis. ${ }^{1}$ Anti-Ro52 has been found in a variety of connective tissue diseases (CTDs) and drawn considerable attention from rheumatologists in recent years. Besides myositis, these autoantibodies have also been reported to be related to ILD in several other CTDs, but with great discrepancy across the studies. ${ }^{2}$ In addition, it remains unclear how the incidence of ILD differs in the presence of anti-Ro52 alone or in combination with anti-Ro60 (Sjogren's syndrome related antigen A), one of the most associated antibodies that may determine anti-Ro52 epitope mapping. ${ }^{3}$

To explore the clinical features of anti-Ro52 and its relationship with anti-Ro60, we retrieved the medical records of 1979 patients tested positive for anti-Ro52 and hospitalised between January 2016 and September 2017 in the Drum Tower Hospital. Both anti-Ro52 and anti-Ro60 were routinely measured using an immunoblotting method (EUROLINE, EUROIMMUN AG, Germany). The majority of our cases were female $(1457,73.6 \%)$ and the average age was $53.0 \pm 16.8$ years old. Totally $1321(66.8 \%)$ patients were diagnosed as having CTDs and 658 (33.2\%) diagnosed as non-CTDs.

Distribution of ILD in patients with various diseases is summarised in table 1. In this cohort, ILD occurred in 37.1\% of anti-Ro52 positive CTD patients and 10.9\% of anti-Ro52 positive non-CTD patients. Among CTDs, idiopathic inflammatory myopathy (IIM) was the most often seen underlying disease (85.4\%), followed by undifferentiated connective tissue disease (UCTD), systemic sclerosis, rheumatoid arthritis (RA) and primary Sjögren's syndrome (pSS). As for systemic lupus erythematosus, only $6.5 \%$ anti-Ro52 positive patients presented ILD, consistent with its low incidence in this prototypic autoimmune disease. $^{4}$

There was no discussion of the difference between antiRo52 single-positive and anti-Ro52/Ro60 double-positive in Sabbagh et al's article. ${ }^{1}$ Previously, it has been implied that the expressions of these two types of autoantibodies were related to different CTDs, ${ }^{5}$ and those with isolated anti-Ro52 were more prone to IIM and inflammatory rheumatism. ${ }^{6}$ Our data showed that the distribution of ILD was also varied between the two groups. The incidence of ILD was increased in both CTD and non-CTD patients with single-positive anti-Ro52 (OR 4.94, p $<0.0001$ and OR 3.41, p $<0.05$ by $\chi^{2}$ and Baptista-Pike analysis). However, compared with those having both anti-Ro52 and anti-Ro60, patients with isolated anti-Ro52 were more likely to develop ILD in RA (OR 6.11), pSS (OR 4.50), polymyositis (PM) (OR 10.00) and UCTD (OR 3.71), but not other CTDs including dermatomyositis (table 1).

In conclusion, our data support that ILD is associated with anti-Ro52, yet the incidence is quite different among various CTDs. For patients with RA, pSS, PM or UCTD, the positivity of anti-Ro52 without anti-Ro60 may indicate the occurrence of ILD.

\section{Si Wu $\odot,{ }^{1}$ Xiaojun Tang, ${ }^{1}$ Liping Wu, ${ }^{1}$ Liang-jing Lu, ${ }^{2}$ Xuebing Feng ${ }^{1}$ \\ ${ }^{1}$ Department of Rheumatology and Immunology, The Affiliated Drum Tower Hospital of Nanjing University Medical School, Nanjing, China \\ ${ }^{2}$ Department of Rheumatology, Renji Hospital, Shanghai Jiaotong University School of Medicine, Shanghai, China}

Correspondence to Dr Xuebing Feng, Department of Rheumatology and Immunology, The Affiliated Drum Tower Hospital of Nanjing University Medical School, Nanjing 210008, China; fengxuebing@hotmail.com

Contributors SW, XT and LW collected data. SW analysed results and drafted the manuscript. L-jLU and XF designed the study. XF supervised the project and edited the manuscript. All authors made substantial intellectual contributions to conception of the work, the interpretation of data and approval of the final manuscript.

Table 1 Distribution of ILD in anti-Ro52 positive patients with various underlying diseases

\begin{tabular}{|c|c|c|c|c|c|c|}
\hline & Total & $\operatorname{Ro}^{2} 2^{+} \operatorname{Ro60}^{-}$ & $\mathrm{Ro}^{2} 2^{+} \mathrm{Ro} 60^{+}$ & OR & $95 \% \mathrm{Cl}$ & $P$ value \\
\hline CTD* & $490(37.1 \%)$ & $352(55.4 \%)$ & $138(20.1 \%)$ & 4.94 & 3.88 to 6.31 & $<0.0001$ \\
\hline IIM ‡ & $123(85.4 \%)$ & $99(88.4 \%)$ & $24(75.0 \%)$ & 2.54 & 0.93 to 6.77 & $>0.05$ \\
\hline$P M$ & $18(66.7 \%)$ & $15(83.3 \%)$ & $3(33.3 \%)$ & 10.00 & 1.42 to 48.96 & $<0.01$ \\
\hline ASS & $21(100.0 \%)$ & $18(100.0 \%)$ & $3(100.0 \%)$ & & & \\
\hline pSS & $236(38.2 \%)$ & $157(57.3 \%)$ & $79(23.0 \%)$ & 4.50 & 3.17 to 6.31 & $<0.0001$ \\
\hline SSC & $15(51.7 \%)$ & $11(57.9 \%)$ & $4(40.0 \%)$ & 2.06 & 0.44 to 8.04 & $>0.05$ \\
\hline UCTD & 49 (64.5\%) & $44(69.8 \%)$ & $5(38.5 \%)$ & 3.71 & 1.16 to 11.61 & $<0.05$ \\
\hline MCTD/overlap syndrome & $7(46.7 \%)$ & $3(30.0 \%)$ & $4(80.0 \%)$ & 0.11 & 0.01 to 1.50 & $>0.05$ \\
\hline Vasculitis & $6(35.3 \%)$ & $5(38.5 \%)$ & $1(25.0 \%)$ & 1.88 & 0.21 to 28.67 & $>0.05$ \\
\hline Non-CTD† & $72(10.9 \%)$ & $68(12.2 \%)$ & $4(3.9 \%)$ & 3.41 & 1.25 to 8.95 & $<0.05$ \\
\hline
\end{tabular}

Data were shown as number (percentage of ILD patients for each disease). Ro52 ${ }^{+}$Ro60 ${ }^{-}$: anti-Ro52 positive and anti-Ro60 negative, Ro52 ${ }^{+}$Ro60 ${ }^{+}$: both anti-Ro52 and anti-Ro60 positive.

*Diagnosis of CTDs was in accordance with the international criteria for classification.

†Patients without a definite CTD during the hospitalisation, of which tumour, infection, ILD and chronic kidney disease were the most common disease types. fincluding DM, PM and ASS.

ASS, anti-synthetase syndrome; CTD, connective tissue disease; DM, dermatomyositis; IIM, idiopathic inflammatory myopathy; MCTD, mixed connective tissue disease; PM, polymyositis; pSS, primary Sjögren's syndrome; RA, rheumatoid arthritis; SLE, systemic lupus erythematosus; SSC, systemic sclerosis; UCTD, undifferentiated connective tissue disease. 
Funding This study was supported by grants from National Key Research and Development Program of China (2017YFC0909003) and National Natural Science Foundation of China (81771745).

Competing interests None declared.

Patient consent for publication Not required.

Provenance and peer review Not commissioned; internally peer reviewed.

(C) Author(s) (or their employer(s)) 2019. No commercial re-use. See rights and permissions. Published by BMJ.

\section{(A) Check for updates}

To cite Wu S, Tang X, Wu L, et al. Ann Rheum Dis Epub ahead of print: [please include Day Month Year]. doi:10.1136/annrheumdis-2019-216372

Received 25 September 2019

Accepted 26 September 2019

Ann Rheum Dis 2019:0:1-2. doi:10.1136/annrheumdis-2019-216372

\section{ORCID iD}

Si Wu http://orcid.org/0000-0001-5371-2384

\section{REFERENCES}

1 Sabbagh S, Pinal-Fernandez I, Kishi T, et al. Anti-Ro52 autoantibodies are associated with interstitial lung disease and more severe disease in patients with juvenile myositis. Ann Rheum Dis 2019:78:988-95.

2 Lee AYS. A review of the role and clinical utility of anti-Ro52/TRIM21 in systemic autoimmunity. Rheumatol Int 2017;37:1323-33.

3 Gkoutzourelas A, Liaskos C, Mytilinaiou MG, et al. Anti-Ro60 seropositivity determines Anti-Ro52 epitope mapping in patients with systemic sclerosis. Front Immunol 2018;9:2835.

4 Hannah JR, D'Cruz DP. Pulmonary complications of systemic lupus erythematosus. Semin Respir Crit Care Med 2019;40:227-34.

5 Murng SHK, Thomas M. Clinical associations of the positive anti Ro52 without Ro60 autoantibodies: undifferentiated connective tissue diseases. I Clin Pathol 2018;71:12-19.

6 Robbins A, Hentzien M, Toquet S, et al. Diagnostic utility of separate Anti-Ro60 and Anti-Ro52/TRIM21 antibody detection in autoimmune diseases. Front Immunol 2019;10:444. 